\title{
Estimate The Scope Of Behavioral Health Need Among Women Beginning Pregnancy Care By An EMR-Based Assessment
}

\section{Shun-ling * and Yu-wenli}

*Corresponding Author: Shun-ling, Department of Mental Health Care,Gaps in Taiwan's mental health care, Taiwan. Email: harazu_21@yahoo.co.jp

Received date: June 02,2018 ;Accepted date : July 13,2018; Published date: Aiugust $29,2018$.

Citation: Shun-ling, Estimate the scope of behavioral health need among women beginning pregnancy care by an EMR-based assessment ,J Psychology and Mental Health Care . Doi: 10.31579/2637-8892/035

Copyright : (c) 2018 Shun-ling. This is an open-access article distributed under the terms of The Creative Commons Attribution License, which permits unrestricted use, distribution, and reproduction in any medium, provided the original author and source are credited.

\section{Abstract \\ Background}

It is well-recognized that behavioral health problems are under detected and undertreated in primary care. Medical settings have been identified as likely settings to detect and address these problems, but any such efforts would require a strategic approach based on needs in the treated population. Obstetric settings are being encouraged to detect and address behavioral health needs.

\section{Methods}

An EMR-based approach for developing an initial estimate of the portion in need, and the range of conditions present, was developed for the obstetric setting. A list was developed of behavioral health diagnoses and prescriptions that could be queried using the electronic medical record (EMR). For diagnoses, the ICD-9 set of "mental disorder" diagnoses (codes 290-319) was used. For prescriptions, a list of behavioral health medications published by NIMH was used, augmented with the behavioral health medications noted in a recent "Top 200" prescription list published by Verispan, a commercial firm.

\section{Results}

Of this cohort of 3,290 women beginning pregnancy care in a one-year time span, this EMR query indicated that 394 (12.0\%) had a behavioral health need; $5.2 \%$ were prescribed a medication alone, $3.1 \%$ had a diagnosis alone, and $3.6 \%$ had both. This is likely an underestimate, but the efficient EMR method serves as a helpful starting place for determining behavioral health needs to be addressed.

\section{Conclusions}

These data indicate that screening for these conditions will likely yield modest, but steady, numbers of patients with behavioral health needs that could be integrated with obstetric care. Readily available data regarding most common diagnoses and most frequently prescribed drugs can be used to develop an initial estimate of the burden of behavioral health need in obstetrics. Other primary care settings could readily replicate this approach as an initial step for addressing behavioral health burden in primary care.

\section{Keywords}

Mental Health, obstetrics, maternal depression, primary care, methodologies, perinatal epidemiology

\section{Introduction}

It is well-recognized that behavioral health problems are under detected and undertreated. Medical settings have been identified as likely settings to detect and address these problems, but any such efforts would require a strategic approach based on needs in the treated population. As in other primary care settings, obstetric settings are increasingly being encouraged to address behavioral healthcare needs [1]. A valid starting place is an estimate of the burden of need and a review of the range of conditions present in a clinic's patient population.

In order to develop such an estimate, an efficient strategy was developed by way of a "meaningful use" approach using the electronic medical record (EMR). This effort was conducted in a United States multi-specialty outpatient medical organization with over 20 outpatient clinics in a large metropolitan area that provides care to an ethnically diverse population of over 400,000 patients. Fifteen of these outpatient clinics include an obstetrics/gynecology service, with over 30 obstetricians who deliver more than 3,000 infants annually.
This obstetrics/gynecology service completed a transition to an EPICbased electronic medical record in 2009, allowing efficient analysis of various patient and treatment characteristics [2].

Evidence notes that many women are taking a prescription medication in the first trimester [3]. Upon discovering pregnancy, women are likely to discontinue a prescription medication they suspect might be harmful $[4,5]$. Patient counseling can provide reassurance, leading to continued maintenance [6].

Depression and stress are associated with negative outcomes including preterm delivery [7] and interference with mother child bonding [8]. Addressing behavioral health requires a healthcare system that can detect behavioral health need and provide access to care [9]. This quality improvement effort closely fits EMR "meaningful use" criteria, a policyfocused healthcare quality improvement trend in the United States. It particularly fits the ability to "generate lists of patients by specific conditions to use for quality improvement, reduction of disparities, research, or outreach," and "identify patient specific education resources [10]." 


\section{Methods}

The approach for developing an initial estimate of behavioral health need in an obstetric setting is reported here. First, a wide definition of behavioral health was adopted [11]. This includes recognized mental health difficulties (depressive disorders, anxiety disorders, and substance use disorders), plus other health issues, such as sleep disorders and chronic pain disorders, that have psychosocial causes and can be effectively treated with psychotherapeutic interventions.

To be efficient, the assessment of behavioral health need was strategically based upon data already recorded in the EMR. This included two types of data: EMR data noting psychiatric diagnoses and prescriptions of medications commonly used for behavioral health conditions. Developing a set of diagnoses was relatively straightforward: the mental disorders set from the ICD-9 [12] was selected: codes 290-319; "Mental Disorders." Because there is a multitude of available prescription medications, it was decided that it would be inefficient to query the use of all medications. Instead, a brief list of commonly prescribed behavioral health medications was developed. Developing a brief list of commonly prescribed medications fit the program planning purpose of identifying common conditions and needs.

Two sources were used to develop this list of medications. First was a list developed by the National Institute of Mental Health [13]. This list includes approved medications organized by the following conditions: schizophrenia, depression, bipolar disorder, anxiety disorders, and ADHD. The second source was published data from the commercial firm Verispan, "Top 200 Brand Drugs by Units in 2007 [14]." This Top 200 list was reviewed and those that had a behavioral health indication were noted. The Verispan list includes an entry for different dosage; the list was collapsed across these for the same medication, relegating multiple listings of any single medication to just one category. Generic and trade names of the same medication were also collapsed into the same category. Each medication was assigned to a leading behavioral health indication, although these medications are often used across indication categories. This process resulted in a list of 50 medications, distributed across the following behavioral health indications: analgesia/pain (15 medications); antidepressant (11); sleep/hypnotic (6); anxiolytic (5); anticonvulsant/bipolar disorder (4); antipsychotic (3); dementia (2); ADHD (2); smoking cessation (1); and menopausal symptoms (1). While it is unlikely to discover pregnant women being prescribed menopausal or dementia medications, this plan was followed in order to illustrate the method for other primary care settings where these might be encountered.

With this list of likely medications and mental disorders, data were extracted from the EMR to create a list of the number of women for the time span, January through December 2009, with any of the ICD mental disorder diagnoses, or prescribed any of the noted medications at the beginning of pregnancy. This study was approved by the Institutional Review Board of the University of Texas Health Science Center-Houston and the Research and Education Committee at Kelsey-Seybold Clinic.

\section{Results and discussion}

Of this cohort of 3,290 insured women beginning pregnancy care in a one-year time span, this EMR query indicated that $394(12.0 \%)$ had a behavioral health need. Table 1 displays the numbers of women with a diagnosis only, a prescription only, or both.

\section{Psychiatric diagnoses}

Among these women beginning pregnancy care, 221 (6.7\%) had a behavioral health diagnosis recorded in the medical chart. Of these, 178 had only one diagnosis, 38 had two, and 5 had three diagnoses. More commonly frequent diagnoses were as follows: $87(2.6 \%)$ had an anxiety/panic diagnosis (ICD code 300); 71 (2.2\%) had a depressive disorder diagnosis (ICD-9 code 311); and 57 (1.7 percent) had a diagnosis of headache (not migraine; code 346). Diagnoses with more than $.1 \%$ occurrence but less than $1.0 \%$ occurrence included: tobacco use (305.1), stress (V62.8), headache (307.8), dysthymia (300.4), and antepartum depression (648).

\section{Behavioral health prescriptions}

Of all women beginning pregnancy care, 293 (8.9 percent) were being prescribed at least one of the surveyed behavioral health medications. The most commonly prescribed category was antidepressants, followed by pain medications. A total of $113(3.4 \%)$ women were prescribed one antidepressant medication, and 3 were prescribed two antidepressants, forming a cohort of 116 women (3.5\%) prescribed an antidepressant when first appearing for pregnancy care. Of all women, 95 (2.9\%) were prescribed a pain medication. There were $21(0.6 \%)$ who were prescribed both an antidepressant and a pain medication.

From the surveyed list of prescription medications, 218 were prescribed a prescription for pain, with hydrocodone being the most common (100; $3 \%)$, followed by acetaminophen $(58 ; 1.8 \%)$, and flexaril $(54,1.6 \%)$. The remaining pain medications were prescribed to less than 1 percent of the cohort. Of the pain medications other than acetaminophen, 16 were prescribed two pain medications, 2 were prescribed three pain medications, and one was prescribed four pain medications. The only sleep medication prescribed was zolpidem, prescribed to $30(0.9 \%)$. Of those prescribed a sleep medication, 20 were not prescribed any antidepressant and 10 were prescribed an antidepressant.

\section{Conclusion}

This EMR chart review methodology efficiently provided an estimate the scope of behavioral health need among women beginning pregnancy care for this healthcare delivery system. This approach may be a sufficient starting point for identifying program planning needs. For a group of pregnant women who are mostly insured, and are beginning pregnancy care in a timely manner (i.e., not beyond first trimester), the predominant conditions requiring program planning seem to be depressive and anxiety conditions, and chronic pain management.

These data indicate that screening for these conditions will likely yield a modest, but steady, numbers of patients with behavioral health needs that could be integrated with obstetric care. What might an obstetric care setting do with such data? Since this study indicated those medications prescribed to approximately one percent or more of a year's cohort of patients, the challenges of other behavioral health conditions can be considered infrequent, and those can be addressed as encountered. In contrast, providers ought to be ready to address the issue of any of the several common medications and conditions.

To be addressed are teratogenic effects, interactions, and patient concerns. Since the medications are known in advance, obstetric settings can prepare and deliver educational information to providers on these medications in advance, rather than a physician seeking out the information when the situation eventually arises. Physicians and others can then be prepared to answer questions regarding safety of these medications in pregnancy. Also, alternatives can be strategically addressed. Some women may be consoled that a medication should be harmless, while others will, nevertheless, want to reduce or discontinue use, and seek alternative strategies. There are psychotherapeutic interventions for all of these conditions (depression, anxiety, sleep, pain, etc.), and so educational materials regarding treatment decision-making can be developed in advance, and psychotherapeutic referral sources can be identified in advance. Physicians and nurses may desire education regarding medication risks, how to screen for these clinical issues, and how to access consultation. The process by which any such behavioral health responses might be delivered, when the need is encountered, can be engineered into the service delivery system in advance.

Following the description provided here, this approach should not be difficult for various healthcare planning teams to adopt. Because new medications come to market each year, and popularity waxes and wanes, a list of commonly prescribed behavioral health medications ought to be updated annually. Healthcare settings are migrating to the ICD-10, and to the DSM-V, so coding of diagnoses will increasingly transfer to the new sets. Older-generation codes will only gradually be replaced and updated. The general approach remains valid, while these specific aspects will need updating. The general approach will serve to indicate behavioral health needs worthy of attention for the purposes of program planning and quality improvement. 
These data provide a conservative estimate, meant for initial strategic efforts to address behavioral health conditions in primary care settings. Mental disorders are likely underdiagnosed as well as underdocumented. Also, unique to the obstetric clinical setting, women may have discontinued a medication upon discovering pregnancy and before attending their first pregnancy appointment, and so may not report these recently discontinued medications when asked about current prescriptions.

Further strategies are required to address those challenges. Obtained EMR prescription results were not very different from a survey of prescriptions during pregnancy. Covering the same time period, the National Birth Defects Prevention Study [11] asked currently or recently pregnant women to report all prescription medications. Of those medications reported by one percent or more of the surveyed women, antidepressants included sertraline (2.22\%), fluoxetine $(1.37 \%)$, and escitalopram (1.04\%). If there were no women taking more than one of these antidepressants, then that would be $4.6 \%$ of the sample taking an antidepressant during pregnancy; that is quite similar to the obtained result of the current study, 3.5\%. Providers should prepare in advance for patient questions and the clinical management challenges of situations likely to be encountered in approximately $5 \%$ or more of their clinical population. This study supports the use of EMR data to detect manage behavioral health conditions in obstetric settings, and this approach may be useful to other primary care settings striving to address the challenge of integrated care.

\section{References}

1. Yonkers KA, Wisner KL, Stewart DE, Oberlander TF, Dell DL, Stotland N, Ramin S, Chaudron L and Lockwood C. The management of depression during pregnancy: a report from the American Psychiatric Association and the American College of Obstetricians and Gynecologists. Obstet Gynecol. 2009; 114:70313.

2. Rowan P, Greisinger A, Brehm B, Smith F and McReynolds E. Outcomes from implementing systematic antepartum depression screening in obstetrics. Arch Womens Ment Health. 2012; 15:115-20.
3. Mitchell AA, Gilboa SM, Werler MM, Kelley KE, Louik C and Hernandez-Diaz S. Medication use during pregnancy, with particular focus on prescription drugs: 1976-2008. Am J Obstet Gynecol. 2011; 205:51 e1-8.

4. Cohen LS, Altshuler LL, Harlow BL, Nonacs R, Newport DJ, Viguera AC, Suri R, Burt VK, Hendrick V, Reminick AM, Loughead A, Vitonis AF and Stowe ZN. Relapse of major depression during pregnancy in women who maintain or discontinue antidepressant treatment. JAMA. 2006; 295:499-507.

5. Petersen I, Gilbert RE, Evans SJ, Man SL and Nazareth I. Pregnancy as a major determinant for discontinuation of antidepressants: an analysis of data from The Health Improvement Network. J Clin Psychiatry. 2011; 72:979-85.

6. Einarson A, Selby P and Koren G. Discontinuing antidepressants and benzodiazepines upon becoming pregnant. Beware of the risks of abrupt discontinuation. Can Fam Physician. 2001; 47:489-90.

7. Straub H, Adams M, Kim JJ and Silver RK. Antenatal depressive symptoms increase the likelihood of preterm birth. Am J Obstet Gynecol. 2012; 207:329 e1-4.

8. Tronick E and Reck C. Infants of depressed mothers. Harv Rev Psychiatry. 2009; 17:147-56.

9. Witt WP, Keller A, Gottlieb C, Litzelman K, Hampton J, Maguire J and Hagen EW. Access to adequate outpatient depression care for mothers in the USA: a nationally representative population-based study. J Behav Health Serv Res. 2011; 38:191-204.

10. Centers for Disease Control and Prevention. Meaningful Use. 2014.

11. Matarazzo JD. Behavioral health and behavioral medicine: frontiers for a new health psychology. Am Psychol. 1980; 35:807-17.

12. American Medical Association. ICD-9-CM Professional for Hospitals (Compact). Eden Prairie, MN: Ingenix; 2010.

13. National Institute of Mental Health. Mental Health Medications. NIH Publication No. 08-3929. Washington, DC: National Institutes of Health, Department of Health and Human Services; 2009.

14. Verispan LLC. Top 200 Drugs for 2007 by Sales. 2014. 